\title{
Formal Distance vs. Association Strength in Text Processing
}

\author{
José Medina Pagola, Ansel Y. Rodríguez González, \\ and Abdel Hechavarría Díaz \\ Advanced Technologies Application Center (CENATAV), 7th Avenue \# 21812 \\ $\% 218$ and 222, Siboney, Playa, Havana City, Cuba \\ \{jmedina, arodriguez, ahechavarria\} @cenatav.co.cu
}

\begin{abstract}
Text information processing depends critically on the proper document representation. Traditional models, like vector space model, have significant limitations because they do not consider semantic relations amongst terms. In this paper we analyze a document representation that uses an association graph scheme model called Global Association Distance Model or GADM, the significance of the formal distance for the association strength, and the application of several distance-strength functions in this model. We evaluate this significance for topic classification tasks.
\end{abstract}

Keywords: Document modelling, Document processing, Document re-presentation.

\section{Introduction}

Nowadays, due to the rapid scientific and technological advances, there are great creation, storage and data distribution capacities. This situation has boosted the necessity of new tools to transform this big amount of data into useful information or knowledge for decision makers. This transformation process is known as Knowledge Discovery in Databases (KDD).

Recent studies and analyses have concluded that complex data require a high number of components to be completely described. This data has to be embedded in high-dimensional spaces (from tens to thousands dimensions). Examples are spectrophotometer data, gene expression data, pictures and texts. In this paper, we focus our analysis on textual data and their representation.

The representation model that is used affects critically almost any text processing task; like information retrieval, classification, clustering, summarization, question-answering, etc. The vector space model is the classic one and by far the most widely used model. Nevertheless, some studies have shown that the weakness of this model is to leave out semantic complexity of the textual data.

As terms appear related to other terms in any document, their meanings strongly depend on the meanings of the surrounding terms; even more, term meanings emerge from mutual sense reinforcement. If we assume that sentences 
are the main semantic unit in texts, then mutual sense reinforcements or associations amongst their terms should be as strong as possible. Nevertheless, it is well known that these reinforcements or associations are feasible in other contexts, such as paragraphs or groups of them. The Global Association Distance Model (GADM) is an association graph scheme that includes this consideration for document representation.

In this paper we analyze the significance of the formal distance for the association strength in GADM, especially in classification tasks using a K-NN classifier. Besides, we evaluate the characteristics of the distance-strength function proposed for this model and propose other functions with better performance.

The basic outline of this paper is as follows. Section 2 is dedicated to related work. In section 3 we describe GADM. Section 4 analyzes several considerations related to formal distance, the association strength, the characteristics of the original distance-strength function and other better functions proposed for GADM, as well as the experimental results obtained.

\section{Related Work}

Document categorization, clustering and information retrieval tasks are often based on good data representation. At a high level, the manipulation of textual data can be described as a series of processing steps that transform the original document representation into another one, simpler and easier to be processed automatically by computers.

This usually involves enriching the document content by adding information, using background knowledge, normalizing terms, etc. At the start of the process, the textual data may exist as a paper, for instance, and the final representation of the preprocessing could be a straight ASCII text enriched with some additional information. This preprocessing final representation is used to represent data in a useful way for computer calculation.

These terms could be organized in different forms but, in general, they are considered as groups or bags of words, usually structured using a vector space model [1]. In this model, term sequences, or their syntactical relations, are not analyzed; therefore, they are considered as unigrams supposing an independence of their occurrences.

In the vector space model, each document is a vector of terms. The values of these vectors could be assumed as weights according the term occurrences in the document or in the document collection, and considering different interpretations [2]: Boolean, Term Frequency and Term Frequency - Inverse Document Frequency.

These vectors of terms are used in a second stage, among other tasks, to analyze the similarities among documents, or groups of them, using different measures as the cosine, applied to the angle between the vectors, defined as [2]:

$$
\operatorname{sim}\left(d_{i}, d_{j}\right)=\cos \left(d_{i}, d_{j}\right)=\frac{\left(d_{i} \bullet d_{j}\right)}{\left\|d_{i}\right\| *\left\|d_{j}\right\|}=\frac{\sum w_{i r} * w_{j r}}{\sqrt{\sum w_{i r}^{2} * \sum w_{j r}^{2}}},
$$


where $d_{i}, d_{j}$ are the vectors of documents $i, j,\|d i\|,\|d j\|$ the norms of the vectors, and $w_{i r}, w_{j r}$ are the term weights in the vectors $d i, d j$, respectively. Other common measures are Dice and Jaccard coefficients.

Alternative approaches to the vector space model are the language models. These consider the probabilities of occurrence of a phrase $S$ in a language $M$, indicated by $P(S / M)$. However, the phrases are usually reduced to one term, assuming again unigrams and independence among them. An example of this model is the Kullback-Leibler Divergence (a variation of the cross-entropy), defined as:

$$
D\left(d_{i} \| d_{j}\right)=\sum P\left(t / d_{i}\right) \log \frac{P\left(t / d_{i}\right)}{P\left(t / d_{j}\right)} .
$$

This expression could be combined in both directions to obtain a similarity measure, as was pointed out by Feldman and Dagan [3].

Other implementation is the proposal of Kou and Gardarin 4. This proposal is a kind of language model, considering the similarities between two documents as:

$$
\operatorname{sim}\left(d_{i}, d_{j}\right)=d_{i} \bullet d_{j}=\sum_{r} w_{i r} w_{j r}+\sum_{r} \sum_{s \neq r} w_{i r} w_{j s}\left(t_{r} \bullet t_{s}\right),
$$

where $w_{i r}$ and $w_{j s}$, using Kou-Gardarin terminology, are the term weights in document vectors $d_{i}, d_{j}$, respectively, and $\left(t_{r} \bullet t_{s}\right)$ is the a priori correlation between terms $t_{r}$ and $t_{s}$. Actually, the authors included in the first part of the expression the self-correlation in $t_{r}$, considering that $\left(t_{r} \bullet t_{r}\right)=1$. The authors propose the estimation of the correlation through a training process. As can be noticed, those correlations express the probabilities $P\left(t_{r}, t_{s} / M\right)$ of phrases containing the terms $t_{r}, t_{s}$ in a language $M$. Besides, that expression could be reduced to the cosine measure (normalized by the length of the vectors) if the term independence is considered and, for that reason, the correlation $\left(t_{r} \bullet t_{s}\right)$ is zero.

Another vector space model is the Topic-based Vector Space Model (TVSM) [5]. The basic premise of the TVSM is the existence of a space $R$ which only has positive axis intercepts. Each dimension of $R$ represents a fundamental topic. It is assumed that all fundamental topics are independent from the others. In this model, each document is represented as a vector of term-vectors; each termvector is a set of weights between the term and the fundamental topics.

The approaches mentioned above are variants of the Generalized Vector Space Model proposed by S.K.M Wong et al. 6. In their work, they expressed that there is no satisfactory way of computing term correlations based on automatic indexing scheme.

We believe that up to the present time that limitation has not been solved yet. Although several authors have proposed different methods of recognizing term correlations in the retrieval process, those methods try to model the semantic dimension by a global distribution of terms, but not with a local evaluation of documents.

In general, it could be assumed that the better the semantic representation of the information retrieved and discriminated, the better this information is mined. 


\section{Global Association Distance Model}

The Association Graph Model assumes that the same term in two documents could designate different concepts [7. Besides, two terms could have different relations, according to the subject of each document, and those relations could exist only in the context of some documents, forming a specific group, and regardless of the relations in a global dimension or language.

In order to model the relation between two terms in a document, the shortest formal (or physical) distance between those terms has been considered. The basic premise of this model can be expressed as follows: Two documents should be closer if the number of common terms is greater and the shortest formal distances among them in each document are similar.

Considering the distance by paragraph, without ignoring the natural cooccurrence when appearing in the same sentence, and considering: $\left(p_{r}, n_{r}\right)$, $\left(p_{s}, n_{s}\right)$, the paragraph and sentence numbers of terms $t_{r}$ and $t_{s}$ respectively, the formal distance between these terms $\left(D_{r s}\right)$ is defined as follows.

$$
D_{r s}=\left\{\begin{array}{cc}
1 & (r=s) \vee\left[\left(p_{r}=p_{s}\right) \wedge\left(n_{r}=n_{s}\right)\right] \\
\left|p_{r}-p_{s}\right|+2 & \text { otherwise }
\end{array} .\right.
$$

Observe that the minimum value of $D_{r s}$, as could be expected, is not zero, but one. This consideration is only a convenient assumption to expressions defined further on.

According to this, a document is modeled by a graph, where the vertices are the distinguished terms and the edges are their relations, weighted by their distances. Notice that this is a fully connected graph, where any term has some relation (stronger or not according to the distance) with the others.

As an improvement to this model, the Global Association Distance Model (GADM) has been proposed [8]. This model uses the preliminary ideas of the classical vector space model proposed by Salton, i.e. to consider " a document space consisting of documents ..., each identified by one or more index terms ... weighted according to their importance" [1]. In GADM, the importance of a term $t_{r}$ in a document $d$ is related to its Global Association Strength $\left(g_{t_{r}}\right)$ and can be calculated as follows.

$$
g_{t_{r}}=\sum_{t_{s} \in d} \frac{1}{\sqrt{D_{r s}}} .
$$

Therefore, the Global Association Distance Model can be defined as a vector space model (VSM) where each term is weighted by their global association strength. Nevertheless, in contradistinction to the original VSM that considers the term relevance by the number of its occurrences in a document, GADM considers the co-occurrences (actually, the association strengths) amongst terms in sentences, paragraphs and so on.

So, a document $d$ can be modelled by a vector of association strengths (6).

$$
\vec{d}=\left(g_{t_{1}}, \ldots, g_{t_{n}}\right)
$$


It is not difficult to understand that the similarity measure between two documents in this model can be calculated by any of the measures defined for the classic vector space model. In that proposal, and also in this work, the cosine measure (1) has only been considered.

\section{Relevance of Formal Distance for the Association Strength}

In order to better understand GADM, consider - as a very simplified examplethe following four terms that appear in the introduction of this proper paper: data, model, classification, and distance. The association strengths amongst these terms are shown in Table 1.

Table 1. Formal distances in the introduction

\begin{tabular}{|l|l|l|l|l|}
\hline \multicolumn{1}{|c|}{$D_{r s}$} & $t_{1}$ & $t_{2}$ & $t_{3}$ & $t_{4}$ \\
\hline$t_{1}$ - data & 1 & 1 & 2 & 3 \\
$t_{2}$ - model & 1 & 1 & 1 & 1 \\
$t_{3}$ - classification & 2 & 1 & 1 & 1 \\
$t_{4}$ - distance & 3 & 1 & 1 & 1 \\
\hline
\end{tabular}

Notice that $D_{12}=1$, because data and model appear together in the third sentence of the third paragraph; $D_{13}=2$, because data and classification appear together on the third paragraph but in different sentences; and $D_{14}=3$, because the third paragraph is the last where data appeared and the fourth paragraph is the first where distance appeared. Also, it can be noticed that the term model has the highest association strength and the term data has the lowest.

Although, in the original work, the formal distance amongst terms was explicitly considered and the authors showed that this single feature can improve the classical VSM with a primary proposal, the relevance of this feature has not been evaluated in all the possible approaches. In the following subsections we will analyze other characteristics of this feature, its significance for this model, and other proposals with better performance.

\subsection{The Distance-Strength Assumption}

The basic assumption of the Global Association Distance Model is that two documents should be closer if the shortest formal distances amongst terms in each document are similar. But also, it indirectly considers the following assumption: The association strength between two terms decreases (increases) as their shortest formal distance increases (decreases). Nevertheless, in the original work, the experimentation was made with two repositories: TREC-5 and Reuters-21758, both with short documents. So, a question is open: Is that assumption true for any distance? Can an upper limit for the distance be considered without losing accuracy? 
In order to find the correct answer, we evaluated one of the short corpora: TREC-5 in Spanish 1. TREC-5 is an AFP news corpus with 695 items published during 1994-1995 and classified in 22 topics. Besides, we prepared a new one with the proceedings of the 18th International Conference on Pattern Recognition (ICPR 2006), held in Hong Kong in 2006. The proceedings have 1130 papers explicitly split into four classes.

All documents were preprocessed and lemmatized with TreeTager 9. In all the evaluations, we have used a $k$-NN classifier with $k=5$. The experimental evaluation was done using $s$-fold cross-validation with $s=10$.

Precision, Recall and $F$-measure are three evaluation measures of performance commonly used. For a single category or topic, these measures can be defined as 10. Precision is the quotient of the correctly assigned and the ones assigned to the category, Recall is the quotient of the correctly assigned and the ones belonging to the category, and $F$-measure is a ponderable representation of both above.

For evaluating the performance average across categories, there are two conventional methods: Macro-averaging and Micro-averaging performance. Macroaveraged performance scores are computed by a simple average of the performance measures for each category. Macro-averaged performance score gives equal weights to every category or topic, regardless of its frequency. Micro-averaged performance scores are computed by first accumulating the corresponding variables in the per-category expressions, and then using those global quantities to compute the scores. Micro-averaged performance score gives equal weights to every document.

Fig. 1 a) and b) show the experimental results for Micro-average with TREC-5 and ICPR-2006 respectively. In this experimentation, we have considered different upper limits of the formal distance, from 1 to 8 , and 10 and 50. So, those terms $t_{s}$, whose distances $D_{r s}$ are greater than each limit, are not considered in formula (5) for term $t_{r}$.
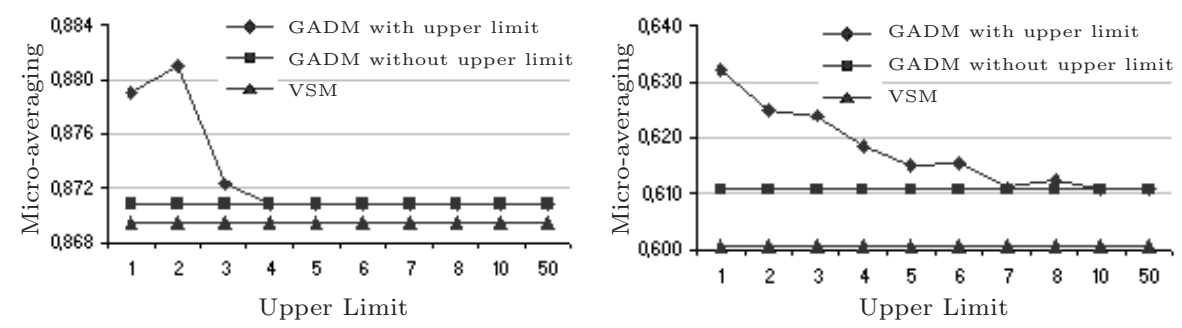

Fig. 1. Results for different upper limits a) TREC-5 b) ICPR-2006

As can be noticed in TREC-5, the best performance is obtained when the formal distance is limited to the same paragraph (upper limit $=2$ ). However, in ICPR-2006 unexpectedly, the best performance is obtained when the upper

${ }^{1}$ http://trec.nist.gov/pubs/trec5 
limit is one, although the results for almost all the upper limits are better than the original results. Nevertheless, as we will show afterwards, it is partially true for GADM.

As a preliminary conclusion, apparently we can obtain better results limiting the formal distance and considering not fully connected graphs, but thresholded graphs with a very small upper limit.

\subsection{The Distance-Strength Function Shape}

In the original work, the global association strength is calculated using formula (5). Nevertheless, other formulae could be used. So, this expression can be generalized in the following way:

$$
g_{t_{r}}=\sum_{t_{s} \in d} d s\left(D_{r s}\right)
$$

where $d s(D)$ is a distance-strength function applied over a formal distance between two terms. In (5) this formula is the function $1 / \sqrt{D}$. However, it has not been proved that this function is the best choice for this model.

The shape of the function is one aspect that should be evaluated first. In this work we have considered three types of functions: convex, linear and concave types.

$$
d s(D)=M^{a}-D^{a} .
$$

The function (8) can be used to model those three types of functions, where $M$ is a superior value of $D$ and $a$ is a parameter that generates the three shapes in the following way:

- If $a<1$, it is a convex function; that is, firstly the strength quickly decreases and at the end it decelerates the decrease with the distance increase.

- If $a>1$, it is a concave function; that is, firstly the strength slowly decreases and then it accelerates the decrease with the distance increase.

- If $a=1$, it is a linear function.

Fig. 2 shows examples of this function with $a=0.5,1$, and 2 .

Fig. (3a) and b) show the behavior of (8) for Micro-average with TREC-5 and ICPR-2006 respectively, taking $M$ as the maximum of paragraph number of each data set. In both figures, the behavior of (8) is compared to the VSM and the
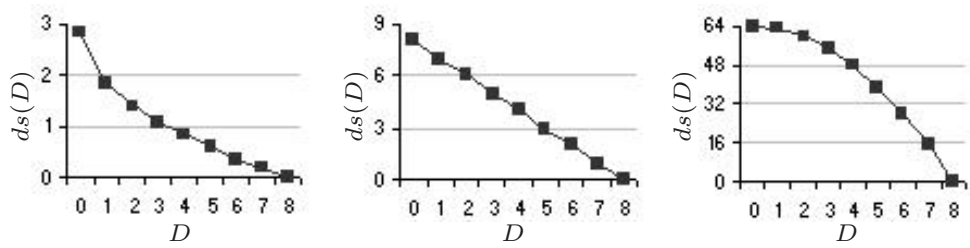

Fig. 2. Example of a convex (left), a linear (center) and a concave (right) function 

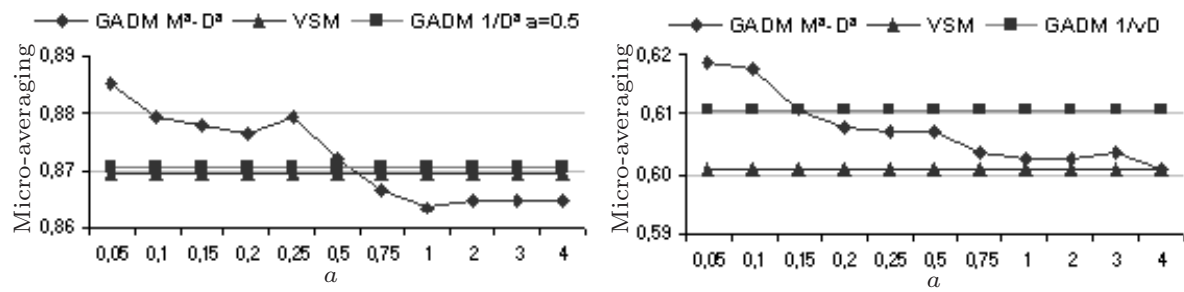

Fig. 3. Behavior of the function with different shapes a) TREC-5 b) ICPR-2006

original GADM formula (5). As can be noticed in both figures, the best results are obtained with convex shapes. It means that, although the formal distance could have a relevant effect, its strength decreases relatively quickly for shortest distances (or nearest paragraphs).

\subsection{The Optimum Distance-Strength Function}

Although the function (8) with a convex shape achieves the best results, it has, as a constraint, to define a constant $M$ which completely depends on document sizes. As an alternative, we can use another family of convex functions, defined in (9), easier to be applied with any document size. Observe that the formula used in (5) is the same as (9) with $a=0.5$.

$$
d s(D)=\frac{1}{D^{a}}
$$

Fig. 4 a) and b) show the behaviors of this new formula for Micro-average with TREC-5 and ICPR-2006 respectively. In both, the behavior of (9) is compared with the VSM and the original GADM formula (5). From this experimentation, we can observe that the original formula (5) is not amongst the best results; moreover, the best results are achieved with formula (9) for values of $a$ in the interval $[4,5]$ in both data sets.

Considering, as a result of the first experimentation made in subsection 4.1 that using an upper limit over the formal distance could improve the behavior, we decided to evaluate formula (9) with different values of $a$ and different upper limits.

Fig. 5 a) and b) show the behaviors of these combinations for Micro-average with TREC-5 and ICPR-2006 respectively. In both, the behavior of (9) is compared with the VSM. Analyzing these results, we can observe that the preliminary conclusion made in subsection 4.1 is partially valid; that is, we can consider not fully connected graphs, or thresholded graphs, with a reasonable upper limit, simplifying the processes associated to GADM. Besides, we can notice that, in TREC-5, the best upper limits are 2 or 3 and in ICPR-2006 could be from 2 to 5 .

As a conclusion, at least in classification tasks with the Global Association Distance Model, we recommend to use the formula (10) as a distance-strength function, considering thresholded graphs with upper limits according to the size 

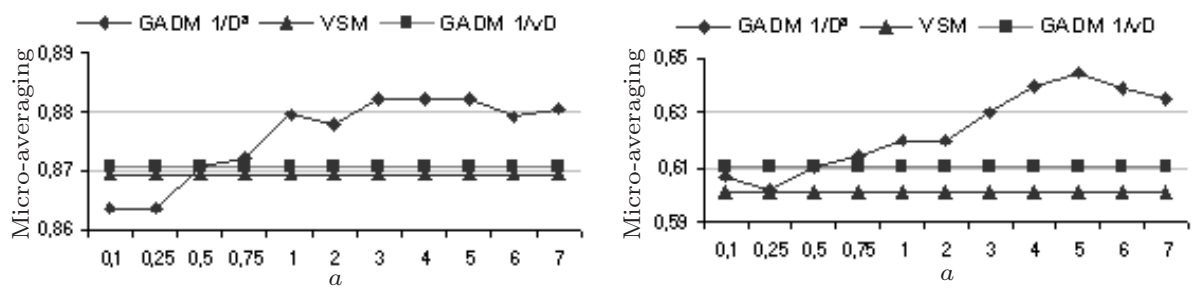

Fig. 4. Behavior of formula (9) a) TREC-5 b) ICPR-2006
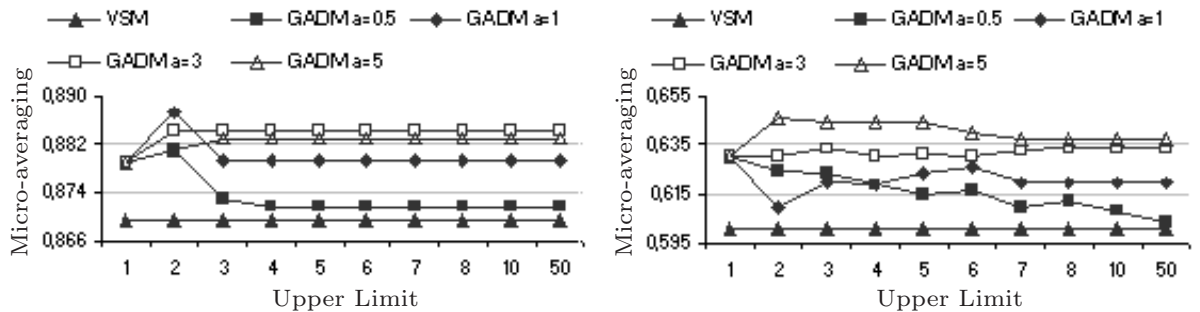

Fig. 5. Behavior of formula (9) and different upper limits a) TREC-5 b) ICPR-2006

of documents to be processed. Also, we can conclude that, although the distances limited to sentences and paragraphs produce the biggest association strengths, and so it is modeled by the best functions, we should take into account the distances to nearest paragraphs because these also contribute, to a lesser extent, to the global improvement.

$$
d s(D)=\frac{1}{D^{5}}
$$

With this new formula and a thresholded graph we obtain the results shown in Table 2 for Micro-average.

Table 2. Results for Micro-average

\begin{tabular}{|c|c|c|c|}
\hline Collection & VSM & GADM, $1 / \sqrt{D}$ & GADM, $1 / D^{5}$ \\
\hline TREC-5 & 0.869401 & 0.870809 & 0.882238 \\
\hline ICPR-2006 & 0.600885 & 0.610619 & 0.645133 \\
\hline
\end{tabular}

Notice that formula (10) represents a $1.48 \%$ improvement in performance compared to VSM and a $1.31 \%$ to the primary proposal in TREC-5, and $7.36 \%$ to VSM and $5.65 \%$ to the primary proposal in ICPR-2006.

\section{Conclusion}

In this paper we have analyzed the significance of the formal distance for the association strength in GADM, especially in classification tasks using a $k$-NN classifier. 
In our experimentation, we showed that GADM not only achieves better results than the classic vector space model in the original formulation, but also in a family of functions with significant improvements.

Besides, we evaluated the characteristics of the distance-strength assumption with different functions and distance thresholds. Also, we proposed a new function with a better performance.

As a future work, it can be analyzed the performance of these considerations with other similarity measures or other forms for modelling the association strength.

\section{References}

1. Salton, G.: The SMART Retrieval System - Experiments in Automatic Document Processing. Prentice-Hall, Englewood Cliffs, New Jersey (1971)

2. Berry, M.: Survey of Text Mining, Clustering, Classification and Retrieval. Springer, Heidelberg (2004)

3. Feldman, R., Dagan, I.: Knowledge Discovery in Textual Databases (KDT). In: KDD 1995, Montreal, pp. 112-117 (1995)

4. Kou, H., Gardarin, G.: Similarity Model and Term Association for Document Categorization. In: Andersson, B., Bergholtz, M., Johannesson, P. (eds.) NLDB 2002. LNCS, vol. 2553, pp. 223-229. Springer, Heidelberg (2002)

5. Becker, J., Kuropka, D.: Topic-based Vector Space Model. In: BIS 2003 (2003)

6. Wong, S.K.M, Ziarko, W., Wong, P.C.N.: Generalized Vector Space Model in Information Retrieval. In: Proc. of the 8th Int. ACM SIGIR Conference on Research and Development in Information Retrieval, p. 11. ACM, New York (1985)

7. Medina-Pagola, J.E., Guevara-Martinez, E., Hernández-Palancar, J., HechavarríaDíaz, A., Hernández-León, R.: Similarity Measures in Documents using Association Graphs. In: Sanfeliu, A., Cortés, M.L. (eds.) CIARP 2005. LNCS, vol. 3773, pp. 741-751. Springer, Heidelberg (2005)

8. Medina-Pagola, J.E., Rodríguez, A.Y., Hechavarría, A., Hernández-Palancar, J.: Document Representation using Global Association Distance Model. In: Amati, G., Carpineto, C., Romano, G. (eds.) ECIR 2007. LNCS, vol. 4425, pp. 565-572. Springer, Heidelberg (2007)

9. Schmid, H.: Probabilistic Part-Of-Speech Tagging Using Decision Tree. In: Proc. of International Conference on New Methods in Language Processing, Manchester, UK (1994)

10. Yang, Y.: An evaluation of statistical approaches to text categorization. Journal of Information Retrieval 1(1/2), 67-88 (1999) 